\title{
Comunicação Interatrial do Tipo Ostium Secundum. Do Tratamento Cirúrgico ao Percutâneo e os Dinossauros do Futuro
}

\author{
Carlos A. C. Pedra, Simone R. F. Fontes Pedra, Valmir Fernandes Fontes
}

São Paulo, SP

A comunicação interatrial tipo ostium secundum corresponde a cerca de $10-12 \%$ de todas as cardiopatias congênitas ${ }^{1}$, sendo uma das lesões mais freqüentes na prática clínica. Por ser uma cardiopatia com hiperfluxo pulmonar, resultante de uma sobrecarga de volume de câmaras direitas, e não de pressão, raramente leva a sintomas significativos na faixa etária pediátrica ${ }^{2}$. O diagnóstico geralmente é sugerido pela presença de sopro cardíaco ejetivo em foco pulmonar e $2^{\text {a }}$ bulha com desdobramento constante e fixo em criança com crescimento pôndero-estatural normal. Classicamente, na radiografia de tórax são encontrados área cardíaca discretamente aumentada, hiperfluxo pulmonar e abaulamento do tronco pulmonar. O eletrocardiograma geralmente mostra sobrecarga de ventrículo direito ${ }^{1,2}$. Entretanto, tais achados podem ser sutis e passarem despercebidos em consultas de rotina, tendo o diagnóstico realizado apenas na adolescência ou idade adulta. Não é infreqüente o seguinte cenário clínico: paciente de 30 anos, do sexo feminino, assintomática, dois partos normais anteriores sem complicações, ausculta cardíaca típica, área cardíaca normal à radiografia de tórax e ecocardiograma mostrando comunicação interatrial do tipo ostium secundum, de cerca de $10 \mathrm{~mm}$, com discreta repercussão hemodinâmica. O que fazer? Qual é a indicação de tratamento em uma paciente adulta assintomática? Qual o método terapêutico de escolha neste caso? Cirurgia X prótese? Como em todos os casos limítrofes em medicina, as respostas não são simples, exigindo reflexão maior.

A pergunta chave, neste caso, em relação à indicação, deve ser: o que é discreta repercussão hemodinâmica? Mesmo pacientes com grande comunicação interatrial e

Instituto Dante Pazzanese de Cardiologia

Correspondência: Carlos A. C. Pedra - Instituto Dante Pazzanese de Cardiologia - Av. Dr. Dante Pazzanese, 500 - 04012-180 - São Paulo, SP Email: vffontes@uol.com.br

Recebido para publicação em 17/7/01

Aceito em 21/1/02 ventrículo direito de dimensões muito aumentadas podem ser assintomáticos e apresentar evolução clínica semelhante ao caso apresentado. No entanto, sabe-se que inúmeros problemas clínicos começam a aparecer após a $3^{\mathrm{a}}, 4^{\mathrm{a}}, 5^{\mathrm{a}} \mathrm{e}$ às vezes até a $6^{\text {a }}$ década, incluindo arritmias secundárias à dilatação atrial direita (fibrilação atrial, flutter, etc), doença vascular pulmonar, disfunção ventricular direita sistólica, disfunção sistodiastólica esquerda (por alteração crônica da interatividade ventricular e anormalidade da contração do septo interventricular) ${ }^{1-5}$. Tais problemas trazem conseqüências clínicas, como palpitações, cansaço, fadiga, limitação das atividades diárias e conseqüente piora da qualidade de vida. Infelizmente, a oclusão tardia do defeito, pelo método cirúrgico ou por prótese, não elimina muitos destes problemas clínicos crônicos, principalmente arritmias e disfunção ventricular ${ }^{1-5}$. Se ainda há shunt atrial esquerdodireito significativo, a oclusão tardia tem algum efeito benéfico, reduzindo o hiperfluxo pulmonar, com impacto na melhoria dos sintomas, como dispnéia, cansaço aos esforços, tosse crônica e bem estar geral ${ }^{6-7}$. Curvas atuariais de sobrevivência mostram uma redução significativa da expectativa de vida em pacientes com comunicação interatrial com repercussão clínica não operadas ${ }^{8}$, o que pode ser revertido quando o defeito é oportunamente tratado ${ }^{3-7}$. Portanto, portadores de comunicação interatrial devem ser tratados com algum grau de repercussão hemodinâmica para evitar problemas clínicos no futuro, além de aumentar a longevidade, mesmo sendo esses pacientes totalmente assintomáticos no momento do diagnóstico. E qual é a linha tênue que às vezes separa o paciente com e sem repercussão hemodinâmica? A resposta não é simples. O maior diâmetro da comunicação interatrial, apesar de importante, não deve ser tomado como parâmetro isolado. As características anatômicas da comunicação interatrial, incluindo seu maior diâmetro, devem ser, sempre que possível, avaliadas pela ecocardiografia transesofágica ${ }^{9,10}$. Mesmo assim, as dimensões do defeito podem apresentar uma variabilidade intra e inter examinador, às vezes surpreendente. Outro aspecto $\mathrm{a}$ 
ser considerado é a variação do diâmetro da comunicação interatrial com o ciclo cardíaco. Pela ecocardiografia bidimensional, não se tem a exata noção desta perspectiva. Reconstruções tridimensionais provenientes de múltiplos cortes transesofágicos mostram uma variação significativa do diâmetro maior da comunicação interatrial e, possivelmente, do shunt $\mathrm{E}-\mathrm{D}$ na sístole e na diástole ${ }^{11,12}$. A complacência do ventrículo direito se constitui em outro possível fator (ainda pouco estudado e entendido) determinante da repercussão clínica do defeito. Por que certos pacientes com comunicação interatrial pequena apresentam shunts significativamente maiores que outros com grandes defeitos? Parte da resposta deve se encontrar na complacência do ventrículo direito e seu efeito sobre a magnitude do shunt atrial, sobretudo na diástole.

Voltando ao caso apresentado, baseando-se nas medidas do ventrículo direito para tomada de conduta clínica, se o ventrículo direito tiver dimensões aumentadas, acima de dois desvios padrão para idade/superfície corpórea, a oclusão estaria indicada a fim de prevenir os problemas clínicos futuros. Apesar da área cardíaca estar normal na radiografia de tórax, lembrando que o perfil esquerdo é a melhor projeção para estimar as dimensões do ventrículo direito, acredita-se que a ecocardiografia transesofágica é mais sensível para definição da repercussão hemodinâmica do defeito ${ }^{9,10,13,14}$. Reconhece-se, também, que até as medidas do ventrículo direito na ecocardiografia transesofágica têm um aspecto subjetivo, o que pode ser minimizado com a experiência do operador. Sinais indiretos de sobrecarga volumétrica do ventrículo direito (como movimentação paradoxal do septo interventricular), também, devem ser considerados. A magnitude do fluxo pulmonar (relação Qp/Qs) pode também ser estimada de forma não invasiva pela medicina nuclear, ajudando na tomada de decisão em casos limítrofes ${ }^{15}$. Relações acima de 1,5 significam shunt E-D significativos.

Qual o melhor método para oclusão? Cirurgia ou prótese? Depende da realidade local de cada instituição, sem dúvida. Os dados de literatura são geralmente derivados de instituições que trabalham em condições próximas das ideais, gerando os "melhores" resultados possíveis, o que nem sempre pode ser transposto para a realidade da maioria dos centros. É raro o encontro de estudos que relatam resultados ruins ou não satisfatórios em relação a um novo procedimento ou técnica. Em outras palavras, a própria literatura é repleta de viés. Os comentários que se sucedem tentam, mas não fogem a esta regra tão comum em cardiologia pediátrica, sendo permeados por uma inevitável visão de quem trabalha com cardiologia intervencionista. Vejamos as vantagens e desvantagens ou limitações de cada método em relação a diversos aspectos.

Intenção de tratar - É inegável que o tratamento cirúrgico pode abordar todo e qualquer tipo de comunicação interatrial tipo ostium secundum, independente da suas dimensões e localização no septo interatrial ${ }^{3}$. Mesmo trabalhando com a prótese Amplatzer, que pode ser aplicada para oclusão de grande comunicação interatrial (até $40 \mathrm{~mm}$ de diâmetro estirado) ${ }^{16,17}$, existem alguns casos que não são fa- voráveis para o implante: comunicação interatrial $>40 \mathrm{~mm}$, comunicação interatrial relativamente grande para as dimensões do paciente (nas quais as dimensões dos átrios não comportariam a presença da prótese) localizações muito excêntricas (bordas superior, inferior ou posterior insuficientes; a medida da borda anterior atrás da aorta geralmente não traz problemas para a Amplatzer) e septos atriais muito complacentes, finos, que não ofereçam muito suporte para a sustentação do dispositivo ${ }^{13}$. Mesmo com essas limitações, estima-se que seja possível ocluir cerca de $70-80 \%$ de todos os defeitos com a prótese Amplatzer ${ }^{13}$. Taxas um pouco menores são vistas para outras próteses como a CardioSEAL, Starflex ou Helex ${ }^{18-21}$. Múltiplos defeitos podem ser abordados com 1 ou 2 próteses, dependendo da distância entre eles ${ }^{22}$.

Necessidade de possuir uma equipe treinada para o implante percutâneo - A atrioseptoplastia (ou rafia) é uma cirurgia relativamente simples do ponto de vista técnico ${ }^{3}$. Para o cateterismo terapêutico, é necessário que o implante seja feito pelo operador mais experiente, com treinamento específico em oclusão de comunicação interatrial ${ }^{23}$. Como o implante da prótese é guiado pela ecocardiografia transesofágica, a presença de um ecocardiografista com experiência em ecocardiografia transesofágica em cardiopatias congênitas, familiarizado com a seleção dos doentes para o procedimento e com os aspectos técnicos do cateterismo, se faz necessário ${ }^{13}$. A inversa também é verdadeira: o intervencionista precisa estar familiarizado e dominar as imagens ecocardiográficas para correta abertura e posicionamento da prótese. A interação ecocardiografista-intervencionista é fundamental para o sucesso do procedimento ${ }^{24}$.

Mortalidade-Na experiência multicêntrica mundial, em mais de 5.000 casos com a Amplatzer (DrZiad Hijazi, comunicação pessoal) e mais de 1.000 com a CardioSEAL/Starflex (Dr Lee Benson, comunicação pessoal) não houve mortalidade. A cirurgia apesar de ser um procedimento seguro apresenta mortalidade de $0,5-2 \%$ nas melhores séries ${ }^{3,25,26}$.

Morbidade-Complicações maiores do procedimento percutâneo são muito raras ${ }^{14,16,18-23}$. Casos de embolização ocorrem infreqüentemente (depende da experiência do operador, tipo de prótese e tamanho do defeito), podendo ser, de forma geral, manejados no próprio laboratório de cateterismo, com resgate dos dispositivos. Perfurações tardias com derrame pericárdico e tamponamento são excepcionais. A ocorrência de acidentes vasculares cerebrais secundários a desprendimento de um fragmento do trombo branco plaquetário, ocorrido nas fases iniciais logo após o implante do dispositivo, é rara, com taxas em torno de $0,4 \%$, correspondendo a ataques isquêmicos transitórios, sem seqüela clínica (Dr Ziad Hijazi, AGA $3^{\text {rd International Sympo- }}$ sium, Strassbourg, França). Episódios embólicos tardios são excepcionais ${ }^{27}$, praticamente não havendo riscos após a endotelização da prótese (em torno de 6-12 meses) ${ }^{28}$. Por outro lado, a cirurgia apresenta uma morbidade significativa ${ }^{26}$. A ocorrência de derrame pericárdico é relativamente comum ( $30 \%$ no Instituto Dante Pazzanese de Cardiologia- 
Dr Tarcisio Almeida - comunicação pessoal), às vezes com tamponamento cardíaco, assim como atelectasias no pós operatório, infecções (na ferida, pneumonia), dor, acidentes vasculares cerebrais em pacientes mais velhos, necessidade de hemoderivados entre outros ${ }^{3,26}$.

Anestesia geral - A anestesia geral deve ser empregada para o tratamento cirúrgico. Devido à necessidade da ecocardiografia transesofágica, o procedimento percutâneo também é realizado sob anestesia geral, com extubação precoce, ainda na sala de cateterismo. Com o advento recente da ecocardiografia intracardíaca, a anestesia geral tem sido dispensada em adultos em alguns centros (Chicago, Toronto ${ }^{29}$. O custo do aparelho e dos transdutores intracardíacos, como o Acuson, introduzidos por bainhas $10 \mathrm{Fr}$, ainda são elevados e constituem-se em uma limitação.

Circulação extracorpórea, anoxia cardíaca eimpacto neurológico - Estudos recentes mostram uma série de efeitos deletérios da circulação extracorpórea sobre o desempenho neurológico, tanto em adultos ${ }^{30}$ como em crianças. Em um estudo realizado no Children's Hospital em Boston, houve um declínio significativo do QI após utilização de circulação extracorpórea para correção de comunicação interatrial. Tal fenômeno não foi observado em um grupo semelhante de pacientes com comunicação interatrial submetido à oclusão percutânea ${ }^{31}$. Apesar desses dados serem preliminares, não é difícil de imaginar que isto possa mesmo ocorrer. Provavelmente, começa-se a aprender e detectar uma série de alterações neurológicas finas após uso de circulação extracorpórea.

Circulação extracorpórea, mediadores da cascata inflamatória e óxido nítrico(NO) - Por inúmeros mecanismos, a circulação extracorpórea ativa uma série de reações da cascata inflamatória, que são deletérios ao desempenho do sistema cardiovascular, causando morbidade significativa no pós-operatório ${ }^{32}$. Tais fenômenos não são observados após tratamento percutâneo da comunicação interatrial(Dr Alan Tarnok, AGA $3^{\text {rd }}$ International Symposium, Strassburgo, França). Além disto, estudos recentes mostram que a oclusão percutânea interfere beneficamente no metabolismo endotelial do $\mathrm{NO}^{33}$, que é sensivelmente afetado pela circulação extracorpórea (Dr Tilman Humpl, comunicação pessoal). Tal aspecto pode ter um impacto significativo em pacientes com algum grau de hipertensão pulmonar, previamente, ao tratamento cirúrgico, aumentando a morbidade pós-operatória.

Tempo de internação - $O$ tratamento percutâneo necessita de menos de $24 \mathrm{~h}$ de internação ${ }^{16,18-23}$. Em alguns países, o procedimento é realizado ambulatorialmente ${ }^{18,19}$. A necessidade de UTI é excepcional. O tratamento cirúrgico requer cerca de 4 dias de internação, com passagem pela UTI de pelo menos $24 \mathrm{~h}^{3,26}$.

Custo do procedimento - Não há muitos estudos dis- poníveis na literatura comparando-se as duas técnicas. Nos EUA, mesmo com o alto custo das próteses, os gastos hospitalares são geralmente menores com o procedimento percutâneo ${ }^{34}$. O tratamento percutâneo teve custos semelhantes ao tratamento cirúrgico por minitoracotomia em um centro na Itália, porém com menor morbidade e tempo de internação ${ }^{35}$. Entretanto, a visão de custos deve levar em conta uma abordagem mais global, que geralmente é negligenciada. Tem-se que considerar nesta equação qual é o custo para a sociedade de um paciente (criança ou adulto) ficar parado por cerca de 15 dias a um mês. Qual é o custo da ausência do paciente (ou pais) no trabalho (produtividade) ou na escola? Com certeza, toda uma rotina familiar é alterada em decorrência da cirurgia. É obrigação do médico, sempre que possível, procurar uma forma de reinserir o paciente na sociedade retomando suas atividades habituais o mais breve possível. Pensamos que o tratamento percutâneo atinge plenamente este objetivo. Além disto, há ainda um outro ponto interessante: qual o custo para outros pacientes cardiopatas? Um centro cardíaco ocupado em locais com poucos centros de referência tem obviamente casos graves na fila (não só complexos, por exemplo: comunicações interventriculares de grande repercussão, cujo o prognóstico é, a princípio, excelente). Qual é impacto que a cirurgia cardíaca para comunicação interatrial tem no retardo da cirurgia para outros defeitos em relação a morbimortalidade desses pacientes? Difícil responder, especialmente em nosso meio.

Eficácia clínica e prevalência de shunt residual - $O$ shunt residual ocorre em torno de 5\% em diversas séries, após um ano do implante da prótese Amplatzer e Starflex ${ }^{16-23,36}$. Comunicações interatriais menores (como no caso hipotético em questão) são as que apresentam as maiores taxas de oclusão ( $>98 \%$ ). Índices um pouco maiores de shunt residual são vistos com a prótese CardioSEAL ${ }^{18,19}$, já não tão utilizada. Independente do dispositivo empregado, a quase totalidade dos shunts residuais tem dimensões "triviais a moderadas" (de 1-4mm), não tendo nenhuma repercussão clínica $^{18-23}$. Ou seja, o paciente está clinicamente tratado, com o ventrículo direito retornando as dimensões normais. O que difere na prática é que esses pacientes com shunts devem receber profilaxia para endocardite, quando necessário. Não se sabe como a presença da prótese pode alterar a história natural benigna em relação à endocardite na comunicação interatrial não operada, que não requer profilaxia $^{37}$. Um ponto importante: o ecocardiografista deve ficar atento para a presença de padrões de fluxo não usuais em volta da prótese. Muitas vezes o observador pode achar que há shunt residual à primeira vista, e uma investigação mais detalhada mostrar apenas uma turbulência discreta de fluxo em volta do dispositivo ${ }^{18}$. Alguns autores preconizam até o uso de microbolhas para aumentar a especificidade do ECO na detecção de shunts adjacentes à prótese ${ }^{38}$. A cirurgia para a comunicação interatrial é vista como um método que proporciona índices de oclusão de 100\%. Quais são as evidências para tal afirmação? A maior parte dos estudos que avaliou os resultados da correção cirúrgica da comu- 
nicação interatrial foi realizada em uma época, quando a ecocardiografia transesofágica com Doppler colorido em aparelhos de alta sensibilidade não estava disponível. Em outras palavras: o rigor metodológico utilizado para avaliar os resultados da oclusão cirúrgica não foi o mesmo do empregado para o tratamento percutâneo. A ecocardiografia transesofágica é realizada após um ano em todos os pacientes submetidos a cirurgia para correção de comunicação interatrial? Acredita-se que não. Ainda que se isto fosse feito, nos surpreenderíamos com a prevalência de shunt residual "silencioso", ou seja, triviais, sem repercussão clínica! E não seria por isto que a cirurgia deixaria de ser eficaz. Não se deve esquecer, que muito do que se sabe atualmente sobre a anatomia tridimensional do septo interatrial in-vivo, além da repercussão hemodinâmica de orifícios de diversos tamanhos, deve-se aos estudos rigorosos aplicados ao procedimento percutâneo ${ }^{39}$. Um único trabalho na literatura, que compara a eficácia das duas técnicas (Amplatzer X Cirurgia), apesar de limitações metodológicas importantes, mostra taxas de oclusão total de $98 \%$ para ambas técnicas, com menor morbidade relacionada ao tratamento percutâneo ${ }^{36}$.

Rápida resposta clínica - A despeito da presença de shunts residuais, existe uma rápida tendência do retorno do ventrículo direito às dimensões normais. Tal fenômeno é documentado pela ecocardiografia seriada pós-implante ${ }^{18,40}$. É muito gratificante notar como a área cardíaca e até a ausculta voltam à normalidade em pouco tempo (às vezes em 24h). Por outro lado, dilatação persistente do ventrículo direito é observada em cerca de $26 \%$ dos casos operados antes da adolescência, mesmo na ausência de shunts residuais ${ }^{25}$. Talvez, uma proteção miocárdica intra-operatória não adequada possa exercer algum papel deletério na redução das dimensões do ventrículo direito.

Cicatriz no septo interatrial e arritmias - Especula-se que o processo de cicatrização e fibrose decorrente da abertura cirúrgica do átrio e da utilização de pontos para oclusão cirúrgica da comunicação interatrial (com ou sem patch) possa levar ao aparecimento de vias arritmogênicas ${ }^{41-43}$. Arritmias variadas, geralmente supraventriculares não são incomuns no pós-operatório imediato e seguimento tardio dos pacientes submetidos a cirurgia. Apesar de não haver estudos comparativos neste sentido, os resultados de longo prazo da oclusão percutânea acenam com a possibilidade deste tipo de abordagem minimizar o trauma no septo interatrial, com taxas menores de arritmias ${ }^{44}$. A ocorrência de arritmias supraventriculares benignas durante e logo após o implante percutâneo é comum ${ }^{18}$. Muito provavelmente, a incidência de arritmias é também (e talvez principalmente) influenciada pelo grau e pelo tempo de dilatação das câmaras direitas.

Cicatriz cirúrgica e satisfação pessoal do paciente A presença de cicatriz decorrente do ato cirúrgico é inevitável, mesmo com a utilização de uma minitoracotomia ${ }^{45}$, que infelizmente não pode ser aplicada a todas as idades. Na verdade, pode-se considerar este tipo de abordagem (minitoracotomia) como um trunfo do procedimento percutâneo. Com isto passou-se a dar uma ênfase maior aos aspectos estéticos após a cirurgia, trazendo benefícios ao paciente. A presença de uma cicatriz cirúrgica pode ter um efeito deletério significativo sobre $\mathrm{o}$ aspecto psíquico do paciente ${ }^{46}$. Tal impacto fica ainda mais crítico, uma vez que a comunicação interatrial acomete uma população predominantemente feminina, que no caso do Brasil, vive em um país ensolarado, onde o culto ao corpo faz parte da cultura. A ocorrência esporádica de depressão psíquica, após uso de circulação extracorpórea, é também um ponto a ser considerado em pacientes adultos. Um outro aspecto que não pode ser esquecido é a satisfação do paciente com o tratamento recebido. É comum preocupar-se com o tamanho da comunicação interatrial, se o paciente tem shunt residual, se recebeu antibióticos para profilaxia para endocardite, etc., mas poucas vezes perguntamos se o paciente está feliz com o que foi feito. Tal questionamento é difícil de ser mensurado objetiva e cientificamente. Estudos que levam em conta e avaliam este tipo de desfecho (outcome) são extremamente bem-vindos. A abordagem percutânea, pelo menos em teoria, tem todas as características de poder proporcionar ao paciente um grau máximo de satisfação pessoal após o procedimento terapêutico. Outro ponto importante, no caso em foco, aplicando a provável história natural da doença e os diferentes métodos terapêuticos disponíveis, de preferência pelos subespecialistas: cirurgião, clínico e intervencionista, qual é a opção do paciente? A obtenção de um consentimento esclarecido para realização de qualquer procedimento, cirúrgico ou não, é dever do médico, o que infelizmente não é prática freqüente.

Seria necessário um estudo multicêntrico randomizado para se comparar as duas técnicas adequadamente. Isto é difícil de ser conseguido em cardiologia pediátrica devido a diversos fatores: número limitado de pacientes em um tempo limitado de estudo, técnicas diferentes em diferentes centros, evolução significativa das técnicas de cateterismo (e porque não cirúrgicas?) em pouco tempo, relutância do paciente em aceitar que caiu no braço cirúrgico do estudo após explicada as vantagens e desvantagens de cada tipo de abordagem, e opiniões pessoais que inevitavelmente permeiam as condutas em centros diferentes. No Instituto Dante Pazzanese de Cardiologia, tem-se optado pela oclusão percutânea da comunicação interatrial com prótese Amplatzer sempre que o caso seja favorável. Infelizmente o SUS ainda não cobre os custos do procedimento, sendo, portanto limitado a pacientes cobertos por seguradoras ou com condições pessoais de arcar com os custos. Nossa experiência é de 53 pacientes, com sucesso no implante em 51 casos. Não houve mortalidade ou complicações maiores. No seguimento, todos os pacientes encontram-se assintomáticos, com atividades normais. Um paciente desenvolveu hipertensão pulmonar três anos após o implante, secundária a tromboembolismo pulmonar devido a uso de anticoncepcional. Em nenhum momento houve detecção de trombo intracardíaco adjacente à prótese nessa paciente, que possuía 
exames ecocardiográficos anteriores absolutamente normais (defeito ocluído, prótese bem implantada, ventrículo direito de dimensões normais, pressões normais em artéria pulmonar). A taxa de oclusão foi de $92 \%$ após um ano do implante, definidas pela ecocardiografia transesofágica. De forma interessante, nos casos com shunt residual (todos $<4 \mathrm{~mm}$ ), o ventrículo direito encontrava-se dentro das dimensões normais, o que eleva a taxa de sucesso clínico para $100 \%$. Tais dados apenas reproduzem a experiência mundial, que é excelente.

Voltando ao caso hipotético, acreditamos que pelos motivos expostos a melhor opção seria a oclusão com dispositivo Amplatzer, uma vez constatado que o ventrículo direito apresenta sobrecarga de volume com dimensões aumentadas, ou seja, há indicação clínica para tal. Uma comunicação interatrial de cerca de $10 \mathrm{~mm}$, geralmente, é muito favorável para oclusão percutânea, não oferecendo o procedimento, dificuldades técnicas, podendo ser realizado em cerca de $1 \mathrm{~h}$ e menos de $10 \mathrm{~min}$ de fluoroscopia.

Fica ainda aqui um questionamento básico, que não foi totalmente esclarecido na literatura. Qual é a idade ideal para o tratamento desta doença? Certamente que não é na idade adulta, pelos motivos já apresentados. Exceto os casos de grande repercussão hemodinâmica, com insuficiência cardíaca e necessidade de terapêutica medicamentosa, que são infreqüentes, devem ser tratados no momento do diagnóstico, mesmo na infância ${ }^{47}$. Na maior parte dos casos, em que há discreta repercussão, o tratamento provavelmente deve ser realizado eletivamente entre os 5-10 anos.

Finalizando, vislumbra-se, ainda, a possibilidade da comunicação interatrial ser tratada clinicamente. Com a evolução da terapia gênica ${ }^{48,49}$, a cirurgia e o implante de próteses para tratamento da comunicação interatrial serão dinossauros do passado em um futuro não distante. Em um primeiro momento, provavelmente ainda na sala de cateterismo, será injetada algum tipo de substância no septo interatrial que o fará crescer com conseqüente oclusão do defeito. Progressivamente, tal substância, ou outra similar, passará a ser aplicada por via endovenosa, intramuscular, ou até mesmo oral. Será interessante o momento em que, olhandose para trás, comentar-se-ão as épocas em que se fazia o coração parar de bater e colocava-se o paciente em um coração-pulmão artificial e que se implantava "discos metálicos" no septo interatrial para fechar um pequeno orifício entre os átrios. A evolução na medicina, dentre uma série de outros fatores, depende do embate entre novas e antigas idéias e da análise crítica dos resultados do nosso árduo trabalho diário. Somente assim é que os novos métodos terapêuticos podem ser estabelecidas definitivamente, tornando realidade os sonhos do passado. King e Mills ${ }^{50}$ que o digam.

\section{Referências}

1. Porter Co-Burn J, Feldt RH, Edwards WD, et al. Atrial septal defects. In: Emmanouilides GC, Riemenschneider TA, Allen HD, Gutgesell HP, editors. Heart Disease in Infants, Children, and Adolescents. Including the Fetus and Young Adults. $5^{\text {th }}$ edition. Baltimore: William \& Wilkins, 1995: 687-703.

2. Rudolph AM. Atrial septal defect and partial anomalous drainage of pulmonary veins. In: Rudolph AM, editor. Congenital Diseases of the Heart: ClinicalPhysiological Considerations. $2^{\text {nd }}$ edition. Armonk, NY: Futura Publishing. 2001: 283-320.

3. Kirklin JW, Barrat Boyes. Atrial septal defect and partial anomalous pulmonary venous connection. In: Kirklin JW, Barrat Boyes, editors. $2^{\text {nd }}$ Edition. Cardiac Surgery. New York: Churchill Livingstone, 1993: 645-74.

4. Morow K, Karp R. Atrial septal defect: lessons from the past, directions for the future. N Engl J Med 1990; 323: 1698-700.

5. McNamara DG, Latson LA. Long-term follow-up of patients with malformations for which definitive surgical repair has been available for 25 years or more. Am J Cardiol 1982; 50: 560-8.

6. Jemielity M, Dyszkiewicz W, Paluszkiewicz L, Perek B, Buczkowski P, Ponizynski A. Do patients over 40 years of age benefit from surgical closure of atrial septal defects? Heart 2001; 85: 300-3.

7. Thilen U, Berlind S, Varnauskas E. Atrial septal defect in adults: thirty-eightyear follow-up of a surgically and a conservatively managed group. Scand Cardiovasc J 2000; 34: 79-83.

8. Campbell M. Natural history of atrial septal defect. Br Heart J 1970; 32: 820-6.

9. Hijazi ZM, Cao Q, Patel HT, Rhodes J, Hanlon KM. Transesophageal echocardiographic results of catheter closure of atrial septal defect in children and adults using the Amplatzer device. Am J Cardiol 2001; 85: 1387-90.

10. Miller-Hance WC, Silverman NH. Transesophageal echocardiography (TEE) in congenital heart disease with focus on the adult. Cardiol Clin 2000; 18: 861-92.

11. Maeno YV, Benson LN, McLaughlin PR, Boutin C. Dynamic morphology of the secundum atrial septal defect evaluated by three dimensional transoesophageal echocardiography. Heart 2000; 83: 673-7.

12. Maeno YV, Benson LN, Boutin C. Impact of dynamic 3D tranesophageal echocardiography in the assessment of atrial septal defects and occlusion by the double-umbrella device (CardioSEAL). Cardiol Young 1998; 8: 368-78.

13. Pedra SRRF, Pedra CAC,Assef JE, et al. Percutaneous occlusion of atrial septal defects. The role of echocardiography. Arq Bras Cardiol 1999; 72: 59-64.
14. Boutin C, Musewe NN, Smallhorn JF, Dyck JD, Kobaiashi T, Benson LN. Echocardiographic follow-up of atrial septal defect after catheter closure by double-umbrella device. Circulation 1993; 88: 621-7.

15. Malcic I, Senecic I, Tezak S, Ivancevic D, Kniewald H. Radioangioscintigraphy and Doppler echocardiography in the quantification of left-to-right shunt. Pediatr Cardiol 2000; 21: 240-3.

16. Berger F, Ewert P, Bjornstad PG, et al. Transcatheter closure as standard treatment for most interatrial defects: experience in 200 patients treated with the Amplatzer Septal Occluder. Cardiol Young 1999; 9: 468-73.

17. Berger F, Ewert P, Nuernberg Jh, Stiller B, Abdul-KhaliqH, Lange PE. Can atrial septal defects larger than $25 \mathrm{~mm}$ effectively and safely be closed by intervention? Cathet Cardiovasc Interv 2001; 53: 134.

18. Pedra CAC, McLaughlin P, Benson LN. The role of CardioSEAL and Star-flex devices in atrial defect occlusion. Curr Interv Cardiol Rep 2000; 2: 274-82.

19. Pedra CAC, Pihkala J, Lee KJ, et al. Transcatheter closure of atrial septal defects using the CardioSEAL implant. Heart 2000; 84: 320-6.

20. Carminati M, Giusti S, Hausdorf G, et al. A European multicentric experience using the CardioSEAL and Starflex double umbrella devices to close interatrial communications holes within the oval fossa. Cardiol Young 2000; 10: 527-33.

21. Latson LA, Zahn EM, Wilson N. Helex septal occluder for closure of atrial septal defects. Curr Interv Cardiol Rep 2000; 2: 268-73.

22. Pedra CAC, Fontes-Pedra SRF, Esteves CA, Assef J, Fontes VF, Hijazi ZM. Multiple atrial septal defects and patent ductus arteriosus: successful outcome using two Amplatzer septal occluders and Gianturco coils. Cathet Cardiovasc Diagn 1998; 45: 257-9.

23. Fontes VF, Pedra CAC, Pedra SRF, et al. Experiência inicial no fechamento percutâneo da comunicação interatrial com a prótese de AMPLATZER.Arq Bras Cardiol 1998; 70: 147-53.

24. Bjornstad PG. Transcatheter closure of atrial septal defects demands co-operation between the interventionist and the echocardiographer. Cardiol Young 2000; 10: 462-4.

25. Meijboom F, Hess J, Szatmari A, et al. Long term follow-up ( 9 to 20 years) after surgical closure of atrial septal defect at a young age. Am J Cardiol 1993; 72: 1431-4.

26. Galal MO, Wobst A, Halees Z, et al. Peri-operative complications following 
surgical closure of atrial septal defect type II in 232 patients - a baseline study. Eur Heart J 1994; 15: 1381-4.

27. Prewitt KC, Gaither NS, Farb A, Worthman DC. Transient ischemic attacks after long-term Clamshell occluder implantation for closure of atrial septal defects. Am Heart J 1992; 124: 1394-7.

28. Kuhn MA, Latson LA, Cheatham JP, et al. Biological responses to Bard Clamshell Septal Occluders in the canine heart. Circulation 1996; 93: 1459-63.

29. Hijazi Z, Wang Z, Cao Q, Koenig P, Waight D, Lang R. Transcatheter closure of atrial septal defects and patent foramen ovale under intracardiac echocardiographic guidance: feasibility and comparison with transesopahgeal echocardiography. Cathet Cardiovasc Interv 2001; 52: 194-9.

30. Newman MF, Kirchner JL, Phillips-Bute B, et al. Longitudinal assessment of neurocognitive function after coronary-artery bypass surgery. N Engl J Med 2001; 344: 395-402.

31. Visconti KJ, Bichell DP, Jonas RA, Newburger JW, Bellinger DC. Developmental outcome after surgical versus interventional closure of secundum atrial septal defect in children. Circulation. 1999; 100(19 Suppl): II145-50.

32. Tarnok A, Hambsch J, Emmrich F, et al. Complement activation, cytokines, and adhesion molecules in children undergoing cardiac surgery with or without cardiopulmonary bypass. Pediatr Cardiol 1999; 20: 113-25.

33. Tworetzky W, Moore P, Bekker JM, Bristow J, Black SM, Fineman JR. Pulmonary blood flow alters nitric oxide production in patients undergoing device closure of atrial septal defects. J Am Coll Cardiol 2000; 35: 463-7.

34. Baker SS, O'Laughlin MP, Jollis JG, Harrison JK, Sanders SP, Li JS. Cost implications of closure of atrial septal defects. Cathet Cardiovasc Interv 2001; 53: 141 .

35. Formigari R, Di Donato RM, Mazzera E, et al. Minimally invasive or interventional repair of atrial septal defects in children: experience in 171 cases and comparison with conventional strategies. J Am Coll Cardiol 2001; 37: 1707-12.

36. Berger F, Vogel M, Alexi-Meskishvili V, Lange PE. Comparison of results and complications of surgical and Amplatzer device closure of atrial septal defects. J Thorac Cardiovasc Surg 1999; 118: 674-8.

37. Bullock AM, Menahem S, Wilkinson JL. Infective endocarditis on an occluder closing and atrial septal defect. Cardiol Young 1999; 9: 65-7.
38. Elzenga NJ. The role of echocardiography in transcatheter closure of atrial septal defects. Cardiol Young 2000; 10: 474-83.

39. Acar P. Three-dimensional echocardiography in transcatheter closure of atrial septal defects. Cardiol Young 2000; 10: 484-92.

40. Veldtman GR, Razack V, Siu S, et al. Right ventricular form and function after percutaneous atrial septal defect device closure. J Am Coll Cardiol 2001; 37 : 2108-13.

41. Bolens M, Friedli B. Sinus node function and conduction system before and after surgery for secundum atrial septal defect: an eletrophysiology study. Am J Cardiol 1984; 53: 1415-20.

42. Medeiros A, Iturralde P, Márquez M, et al. Permanent rhythm and conduction disorders in patients surgically treated for atrial septal defect. Arch Inst Cardiol Mex 2000; 70: 46-54.

43. BergerF, Vogel M, Kramer A, ETAL. Incidence of atrial flutter/fibrillation in adults with atrial septal defect before and after surgery. Ann Thorac Surg 1999; 68: 75-8.

44. Schenck MH, Sterba R, Foreman CK, Latson LA. Improvement in noninvasive electrophysiologic findings in children after transcatheter atrial septal defect closure. Am J Cardiol 1995; 76: 695-8.

45. Bichell DP, Geva T, Bacha EA, Mayer JE, Jonas RA, del Nido PJ. Minimal access a approach for the repair of atrial septal defect: the initial 135 patients. Ann Thorac Surg 2000; 70: 115-8.

46. Giannotti A. Sentimentos, atitudes e problemas emocionais dos cardiopatas congênitos. In: Giannotti A, editor. Efeitos Psicológicos das Cardiopatias Congênitas. Psicologia em Instituições Médicas. São Paulo: Lemos Editorial, 1996: 109-56.

47. Castaneda AR, Jonas RA, Meyer JE, Hanley FL. Atrial septal defect. In Castaneda AR, Jonas RA, Meyer JE, Hanley FL, editors. Cardiac Surgery of the Neonate and Infant. Philadelphia: WB Saunders, 1994: 143-55.

48. Mason CA, Bigras JL, O'Blenes SB, et al. Gene transfer in utero biologically engineers a patent ductus arteriosus in lambs by arresting fibronectin-dependent neointimal formation. Nat Med 1999; 5: 141-2.

49. Chien KR, Shimizu M, Hoshijima M, Minamisawa S, GraceAA. Towardmolecular strategies for heart disease - past, present, future. Jpn Circ J 1997; 61: 91-118.

50. King TD, Thompson SL, Steiner C, Mills NL. Secundum atrial septal defect: nonoperative closure during cardiac catheterization. JAMA 1976; 235: 2506-9.

\section{ERRATA}

No artigo “Alterações Genéticas e Colesterolemia: Recentes Estudos Brasileiros”, de Forti e cols., publicado em Arq Bras Cardiol 2003; 80(5): 565-71, à p. 567, $1^{\circ}$ coluna, $4^{\circ}$ parágrafo, $10^{\circ}$ linha, onde se lê "figura 1", leia-se "tabela I"; à p. 569, no título da tabela, onde se lê "normolipidêmicos hipercolesterolêmicos", leia-se "normolipidêmicos e hipercolesterolêmicos"; à p. 566, $2^{\circ}$ coluna, $1^{\circ}$ parágrafo, última linha, onde se lê "e4", leia-se "alelo \&4";

As abreviaturas utilizadas foram: CT (colesterolemia total), TG (trigliceridemia), HDL-c (colesterol ligado à lipoproteína de baixa densidade), LDL (lipoproteína de baixa densidade), CT/HDL-c (relação entre CT e HDL-c, índice de Castelli 1). 